

\section{National clinical practice guidelines: a logical advancement of the profession}

Peter Jurkovsky LLM, LLB(Hons), DipParaSc, GradDipLegalPrac, GradCertHighEd is a paramedic, educator and lawyer. $\mathrm{He}$ is President and Board Director of Paramedics Australasia and Chair of the Australian National Registration Working Group.

Clinical practice guidelines (CPGs) are a particularly important element of paramedicine because of our culture of mandatory adherence to this fundamental resource to guide autonomous practice. As the profession evolves it is therefore a logical advancement to develop then implement a national set of CPGs.

The Paramedics Australasia (PA) charter decrees that PA 'provides a respected voice in determining how changes in health service provision, legislation and clinical practice are shaped and implemented to enhance the quality of patient care'. We do this by representing the interests of practitioners on matters relating to:

- $\quad$ health policies that affect the access to and delivery of front-line primary and emergency care

- $\quad$ the professional standards of education, training, and continuing professional development

- the funding, service standards, quality and accreditation of emergency service providers

- $\quad$ the registration of practitioners and the regulatory framework of paramedic practice

- $\quad$ the fostering of best practice through research into out-of-hospital and emergency medical care

- $\quad$ enhanced communication and interaction between paramedics and other health and allied health professionals.

In line with this charter and coinciding with the commencement of national registration later this year, PA is eager to drive a conversation around, and the development of, national CPGs.

To this end, in 2016 PA asked Marc Colbeck to oversee a project to review CPGs. He invited Sonja Maria to partner with him (both are paramedics, academics and PhD candidates focussing their studies on paramedic CPGs). Their comprehensive introductory article, 'A comparative taxonomy of Australasian paramedic clinical practice guidelines' is published in this issue of the AJP and we trust it will stimulate a much broader discussion on the need for national CPGs and the manner in which the project should proceed (1).

Paramedics Australasia believes that a national set of CPGs is a logical advancement of the profession in Australia with multiple benefits - not the least being enhanced public safety. A national set of CPGs would align paramedic practice between each jurisdiction to ensure that ultimate best practice is standard across Australia. The end-user (the patient) is the one who will benefit most from a consistent evidence-based approach to paramedic practice. Education standards and curricula can be based on uniform teaching from the same CPGs regardless of state/university, leading to consistent expectations. National CPGs would bring Australia to the forefront of ambulance practice worldwide, matching the benchmarks created by jurisdictions such as the United Kingdom. Clinical governance could be aggregated across each ambulance service promoting collaboration and sharing of ideas. It would also create greater flexibility for paramedics to move between employers post-registration with clearly defined scopes of practice. These are just some of the potential benefits that could be gained from this initiative with the overarching objective of improved patient outcomes.

Most importantly, PA sees this project as requiring a whole-of-industry approach and is eager to engage with all stakeholders in reaching this extremely worthwhile and achievable goal. I recommend the Colbeck and Maria article to you, and look forward to much engagement and discussion as the project progresses.

Peter Jurkovsky

President and Board Director of Paramedics Australasia

Chair of the Australian National Registration Working Group

\section{Reference}

1. Colbeck M, Maria S. A comparative taxonomy of Australasian paramedic clinical practice guidelines. Australasian Journal of Paramedicine 2018;15. 